\title{
Hidroxietil Celulose Enxertada com Poliéteres
}

\author{
Anelise M. Regiani, Agnieszka Pawlicka, A. Aprigio S. Curvelo, Alessandro Gandini e Jean-François Le Nest
}

Resumo: Foram preparadas amostras de hidroxietil celulose (HEC) enxertada com poliéteres para aplicação como eletrólitos sólidos poliméricos. Amostras comerciais de HEC foram caracterizadas por ${ }^{1} \mathrm{H}$ e ${ }^{13} \mathrm{C}$ RMN, FTIR e viscosimetria, visando a determinação dos respectivos graus de substituição molar (MS), grau de substituição (DS) e grau de polimerização (DP). Amostras comerciais de diaminas de poli(óxido de etileno) (POE) e poli(óxido de propileno) (POP) foram também caracterizadas antes de serem convertidas nos correspondentes diisocianatos. As redes foram obtidas através de reações de condensação das HEC com os diisocianatos e caracterizadas por FTIR, UV-Vis, difração de raios-X e análises térmicas, a fim de definir suas $\mathrm{Tg}$.

Palavras-chave: Redes de poliéteres, hidroxietil celulose, enxertia, eletrólitos sólidos poliméricos

\section{Introdução}

O desenvolvimento de novos materiais sólidos para aplicação em eletrólitos permite a criação de modernos sistemas de geração e armazenamento de energia ${ }^{[1]}$. Dentre estes materiais os eletrólitos sólidos poliméricos, geralmente elastômeros contendo íons, representam uma alternativa promissora para a substituição dos eletrólitos líquidos e cristais inorgânicos utilizados em baterias, sensores e dispositivos eletrocrômicos ${ }^{[2]}$.

Materiais baseados em estruturas de poli(óxido de etileno) têm sido extensivamente estudados ${ }^{[3,4]}$, no entanto estes materiais apresentam algumas características que limitam o seu desempenho, decorrentes da estrutura linear e regular destas macromoléculas ${ }^{[4]}$. Dentre estes problemas está a tendência à cristalização, uma vez que a cristalinidade da matriz polimérica é um grande obstáculo ao fenômeno de condução iônica. Uma forma de resolver este tipo de inconveniência é a preparação da matriz polimérica com a estrutura modificada através da reação com moléculas pequenas ou oligômeros ${ }^{[4]}$, diminuindo a ordem local e eliminando a tendência à cristalização. Um exemplo desta reação é a condensação de dióis ou trióis de oligoéteres com isocianatos polifuncionais, obtendo-se materiais com ligações cruzadas, que podem eliminar os problemas devido ao fluxo viscoso associado às estruturas termoplásticas $^{[2]}$. A redução da cristalinidade pode ser também conseguida através de reações de enxertia como, por exemplo, verificada nos derivados de celulose como a própria HEC ${ }^{[5]}$. Uma grande variedade de redes podem ser formadas através da variação do tamanho das macromoléculas e da

Anelise M. Regiani, Agnieszka Pawlicka, A. Aprigio S. Curvelo, Departamento de Físico-Química, Instituto de Química de São Carlos - USP, Av. Dr. Carlos Botelho 1465, C.P. 780, 13560-970 São Carlos - SP. Agnieszka@iqsc.sc.usp.br; Alessandro Gandini, Jean-François Le Nest, Matériaux Polyméres, Ecole Française de Papéterie et des Industries Graphiques - INPG, BP 65, 38402 St. Martin d'Hères, França 
funcionalização do isocianato. É descrito na literatura o estudo sistemático destes eletrólitos ${ }^{[2]}$ estabelecendo uma relação entre a estrutura da rede e suas propriedades, tais como $\mathrm{T}_{\mathrm{g}}$, comportamento dinâmico-mecânico, condutividade iônica e estabilidade eletroquímica entre outras.

Com o conhecimento dos parâmetros de estabilidade e condutividade destes eletrólitos sólidos torna-se interessante o estudo de outras questões, como a capacidade de obtenção de filmes muito finos, para aplicação em artefatos onde esta característica é imprescindível, como por exemplo, em dispositivos eletrocrômicos. Alguns polissacarídeos e seus derivados possuem grande capacidade para serem processados na forma de filmes finos. Para este fim, os derivados de celulose representam uma classe promissora $^{[6,7]}$, pois os filmes obtidos a partir destes polímeros aliam boas propriedades mecânicas e filmógenas, além de serem materiais economicamente viáveis devido tanto à grande disponibilidade de celulose na natureza como também ao seu caráter renovável ${ }^{[6]}$. Observando este contexto, melhores resultados de condução são obtidos com a modificação da estrutura dos derivados através de reações de enxertia e entrecruzamento (total ou parcial) com introdução posterior de íons lítio ${ }^{[7]}$.

Este trabalho tem como objetivo principal apresentar a técnica de obtenção de filmes de hidroxietil celulose enxertada com cadeias de poliéteres visando sua possível utilização como eletrólitos sólidos poliméricos.

\section{Experimental}

\section{Caracterização de HEC}

As amostras de hidroxietil celulose (HEC), gentilmente cedidas pela UNION CARBIDE do Brasil sob os nomes QP09H, QP300H, QP4400, QP15MH e QP30MH, foram caracterizadas através da espectroscopia na região do infravermelho (FTIR), ressonância magnética nuclear de hidrogênio e de carbono ( ${ }^{1} \mathrm{H}-\mathrm{RMN}$ e $\left.{ }^{13} \mathrm{C}-\mathrm{RMN}\right)$, viscosimetria e difração de raios-X.

Os espectros de FTIR foram obtidos utilizandose aparelho BOMEM modelo MB-102. As amostras de HEC antes de prensadas com $\mathrm{KBr}$ na forma de pastilhas na proporção 150:1, foram cuidadosamente secas em sistema do tipo Abderhalden, sob refluxo com metanol durante $24 \mathrm{~h}$.
Os espectros de ${ }^{1} \mathrm{H}-\mathrm{RMN}$ foram obtidos com espectrômetro BRUCKER AC200 (200MHz) a $34,5^{\circ} \mathrm{C}$ em tubo de $5 \mathrm{~mm}$ de diâmetro a partir de amostras de HEC, a 20\%, dissolvidas em ácido clorídrico $^{[8]}$.

Os espectros de ${ }^{13} \mathrm{C}-\mathrm{RMN}$ das amostras de HEC foram obtidos com espectrômetro BRUCKER AC50 $(50 \mathrm{MHz})$ a $75^{\circ} \mathrm{C}$ em tubo de $10 \mathrm{~mm}$ de diâmetro; a concentração da amostra foi de $200 \mathrm{mg} / \mathrm{mL}$ em DMSO- $d_{6}{ }^{[9]}$.

As massas molares das HEC foram determinadas através das medidas de viscosidade de soluções do polissacarídeo a várias concentrações ${ }^{[10]}$, realizadas em viscosímetro capilar do tipo Ubbelohde submerso em banho termostático a $25^{\circ} \mathrm{C}$.

As análises térmicas DSC foram feitas com aparelho DSC 2910 - Differencial Scanning Calorimeter - TA Instruments usando-se a razão de aquecimento de $10^{\circ} \mathrm{C} / \mathrm{min}$ sob atmosfera de nitrogênio ${ }^{[11]}$.

A análise por difração de raios-X foi feita com um Difratômetro Universal de Raios-X URD-6, Carl Zeiss Jena, a potência de $\mu=40 \mathrm{kV} / 20 \mathrm{~mA}$ e irradiação $\mathrm{CuK} \alpha \operatorname{com} \lambda=1,542 \AA$.

\section{Caracterização das aminas e isocianatos}

As aminas comerciais Jeffamine ED600 e Jeffamine D400 (FLUKA) e os respectivos isocianatos foram caracterizados por FTIR, colocando-se uma gota de amostra sobre pastilha de $\mathrm{KBr}$. Os espectros de ressonância magnética $\left({ }^{1} \mathrm{H} \mathrm{e}{ }^{13} \mathrm{C}\right)$ foram registrados a temperatura ambiente utilizando-se como solvente DMSO- $d_{6}$ para as aminas e $\mathrm{CDCl}_{3}$ para os isocianatos. As massas molares das aminas foram determinadas por titulação com solução padrão de ácido clorídrico $0,1 \mathrm{~N}^{[12]}$.

\section{Síntese dos diisocianatos}

Os diisocianatos de poli(óxido de etileno) e poli(óxido de propileno) foram sintetizados a partir das respectivas Jeffaminas comerciais seguindo método experimental descrito na literatura ${ }^{[13-15]}$.

\section{Entrecruzamento e caracterização das redes}

Misturou-se uma solução de HEC em DMAc e uma solução de diisocianato em DMAc na presença do catalisador dilaurato de dibutilestanho (Aldrich) dentro de câmara seca sob atmosfera de $\mathrm{N}_{2}$. O filme foi moldado em placa de vidro e, $48 \mathrm{~h}$ após sua formação, foi seco em estufa a $40^{\circ} \mathrm{C}$. Os filmes obti- 
dos foram caracterizados através de análise de calorimetria exploratória diferencial (DSC), com equipamento SHIMADZU DSC-50, no intervalo de temperatura de -100 a $200^{\circ} \mathrm{C}$ sob fluxo de nitrogênio $(20 \mathrm{~mL} / \mathrm{min})$. A primeira corrida foi realizada a uma taxa de aquecimento de $20^{\circ} \mathrm{C} / \mathrm{min}$ e a segunda, utilizada na determinação, de $\mathrm{T}_{\mathrm{g}}$, a $10^{\circ} \mathrm{C} / \mathrm{min}$.

Os filmes foram também caracterizados através de técnicas de FTIR (aparelho Perkin-Elmer PARAGON 1000), UV-Vis (Hitashi U-350) e de difração de raios-X.

\section{Resultados e Discussão}

\section{Caracterização de HEC}

O grau de substituição molar (MS) das amostras de HEC foram obtidos dos correspondentes

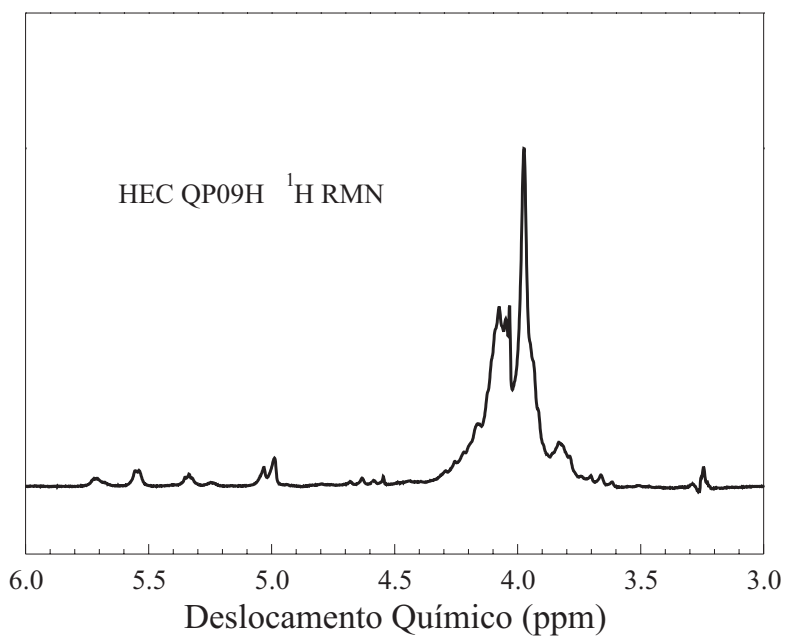

(a)

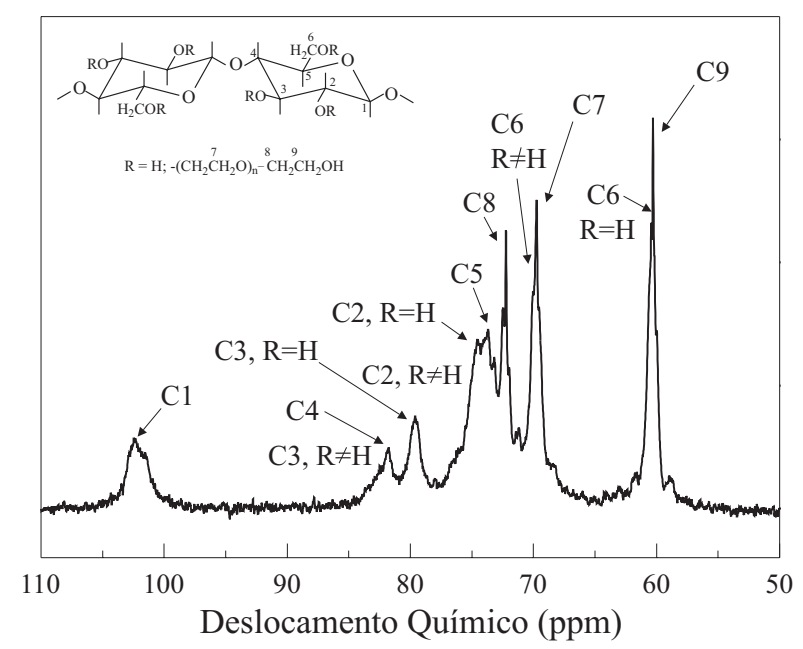

(b)

Figura 1. Espectros da amostra de HEC QP09H (a) ${ }^{1} \mathrm{H}-\mathrm{RMN}$ e (b) e ${ }^{13} \mathrm{C}-\mathrm{RMN}$.
Tabela 1. Características das amostras de hidroxietil celulose (HEC).

\begin{tabular}{cccc}
\hline HEC & MS & DS & DP \\
\hline QP09H & 1,22 & 0,75 & 260 \\
QP300H & 0,58 & 0,34 & 850 \\
QP4400 & 1,35 & 0,88 & 1840 \\
QP15MH & 1,72 & 1,01 & 2450 \\
QP30MH & 0,91 & 0,54 & 2480 \\
\hline
\end{tabular}

espectros de ${ }^{1} \mathrm{H}-\mathrm{RMN}$ (Figura 1a), a partir das áreas referentes aos picos característicos de 3,5 a 4,4 ppm (contribuição de 6 hidrogênios do anel de anidroglicose) e de 4,3 a 5,8 ppm (contribuição de 4 hidrogênios do grupo substituinte hidroxietil ${ }^{[8]}$. O grau de substituição (DS) foi determinado a partir dos valores de MS e da razão das áreas relativas aos sinais de ${ }^{13} \mathrm{C}-\mathrm{RMN}$ de 69,72 e $60,03 \mathrm{ppm}$ (Figura 1b) ${ }^{[9]}$. O grau de polimerização (DP) das diferentes amostras foi calculado a partir de medidas de viscosidade, determinando-se as correspondentes viscosidades intrínsecas e aplicando-se a equação de Mark-Houwick-Sakurada. Foram utilizados valores de $K=1,1 * 10^{-2}$ e $\alpha=0,87$ obtidos da literatura $^{[11]}$. Os valores de DS, MS e DP são valores médios e estão apresentados na Tabela 1, em ordem crescente de DP. Todas as amostras estudadas possuem valores de DS igual ou inferior a 1 e apresentam uma boa relação linear com os respectivos valores de MS (Figura 2). Esta linearidade (no intervalo estudado) é conseqüência do método industrial de obtenção da HEC, o qual consiste em uma primeira etapa de ativação da celulose por hidróxido de sódio e posterior adição do óxido de etileno $^{[16]}$. Vale a pena ressaltar, que esta depen-

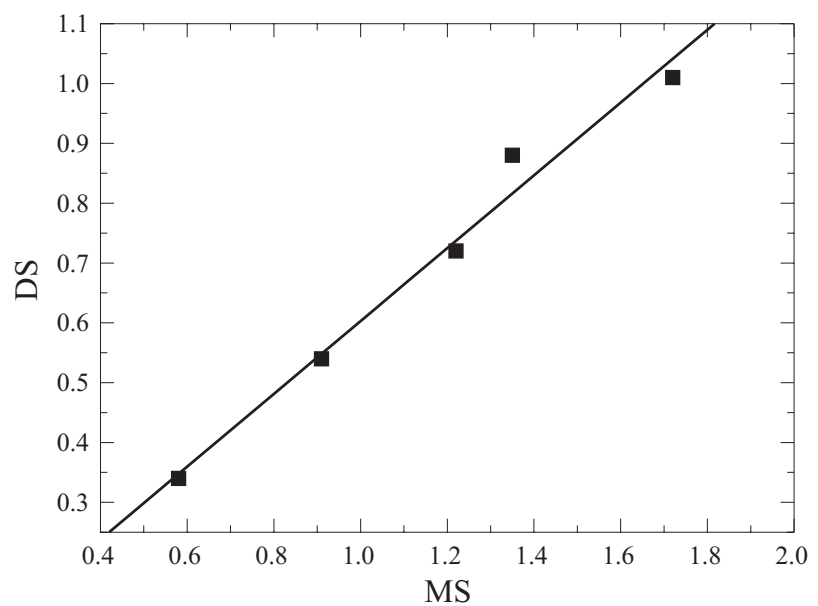

Figura 2. Grau de substituição (DS) versus grau de substituição molar (MS) para amostras comerciais de hidroxietil celulose (HEC). 


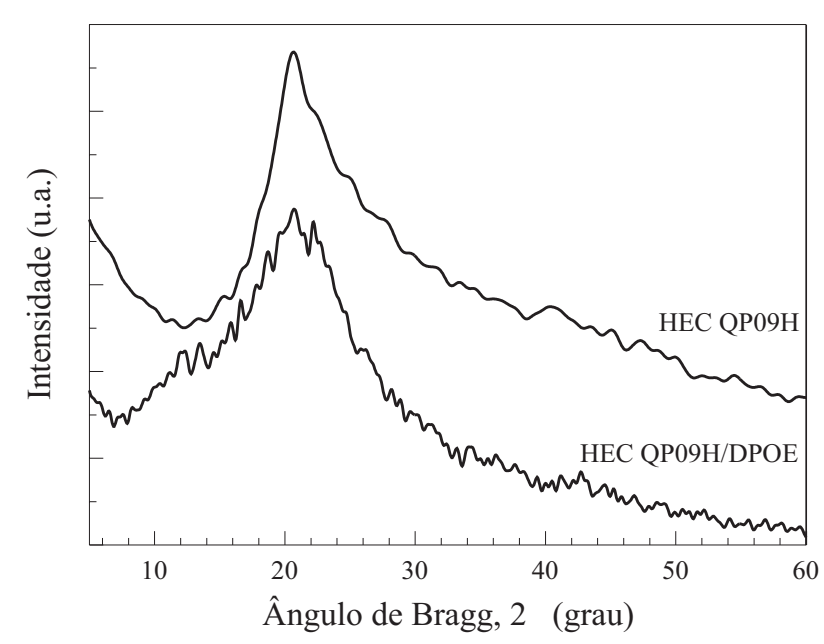

Figura 3. Difractogramas de raios-X das amostras de HEC QP09H e QP09H/DPOE.

dência linear é limitada, já que poderiam ser substituídos no máximo três grupos $\mathrm{OH}$ presentes na unidade de anidroglicose, levando ao valor de $\mathrm{DS}=3$. Entretanto o valor de MS poderia ser infinito por causa dos grupos $\mathrm{OH}$ dos substituintes serem sempre livres para reação com o óxido de etileno. Os valores de DP obtidos, ainda que elevados para algumas amostras, concordam com dados da literatura ${ }^{[11]} \mathrm{e}$, como esperado, não mostram qualquer relação com os valores de DS e MS, indicando que o tamanho da cadeia de celulose não afeta o rendimento da reação de oxietilação nem a distribuição dos grupos substituintes.

$\mathrm{Na}$ análise dos difratogramas de raios- $\mathrm{X}$ das amostras de HEC notou-se a presença de banda larga centrada a ângulo de Bragg a $2 \theta=20^{\circ}$ (Figura 3) indicando a existência de regiões cristalinas. Esta única banda, encontrada na mesma região do principal pico observado em amostras de celulose $\mathrm{e}^{[5]}$, e comparada com os picos geralmente observados indica a diminuição da cristalinidade da HEC em relação a celulose. Não foi observada a presença de nenhum outro pico cristalino caracterizando, portanto, a predominância do estado amorfo das amostras.

\section{Caracterização das aminas e dos isocianatos}

Os espectros de ressonância magnética nuclear $\left({ }^{13} \mathrm{C}\right)$ dos isocianatos preparados apresentam a absorção típica do grupo NCO em 125 ppm e os espectros de infravermelho (FTIR) contém a forte absorção em $2250 \mathrm{~cm}^{-1}$ característica deste grupo funcional. As
Tabela 2. Características das aminas de poli(óxido de etileno) e poli(óxido de propileno) e respectivos diisocianatos.

\begin{tabular}{lc}
\hline \multicolumn{1}{c}{ Amina } & $\begin{array}{c}\text { Massa molar } \\
(\mathbf{g} / \mathbf{m o l})\end{array}$ \\
\hline $\begin{array}{l}\text { O,O'-bis(2-aminopropil)polietilenoglicol 500 } \\
\text { (JEFFAMINE ED600) }\end{array}$ & 660 \\
$\begin{array}{l}\text { O,O'-bis(2-aminopropil)polipropilenoglicol 300 } \\
\text { (JEFFAMINE D400) }\end{array}$ & 450 \\
\hline \multicolumn{1}{c}{ Isocianato } & $\mathbf{\%} \mathbf{N C O}$ \\
\hline $\begin{array}{l}\text { O,O'-bis(2-isocianopropil)polietilenoglicol 500 } \\
\text { (DPOE) }\end{array}$ & 8,4 \\
$\begin{array}{l}\text { O,O'-bis(2-isocianopropil)polipropilenoglicol } \\
\text { 300 (DPOP) }\end{array}$ & 11,6 \\
\hline
\end{tabular}

massas molares das aminas estão apresentadas na Tabela 2 juntamente com o teor de grupos $\mathrm{NCO}$ dos isocianatos preparados. Os teores de $\mathrm{NCO}$ obtidos $8,4 \%$ e $11,6 \%$ correspondem a um percentual de conversão de funções de amina primária de $61,3 \%$ e $66,7 \%$, respectivamente.

\section{Propriedades das redes}

Para avaliar a influência dos parâmetros (DS, MS e DP) da HEC sobre as características das redes, as amostras deste polissacarídeo foram submetidas às reações de entrecruzamento com a mesma proporção de diisocianato. A relação utilizada foi de 0,6 funções NCO para cada função hidroxila, obtendo-se uma estrutura tridimensional como apresentada na Figura 4, para o uso do macrodiisocianato de óxido de oligoetileno (DPOE, Tabela 2).

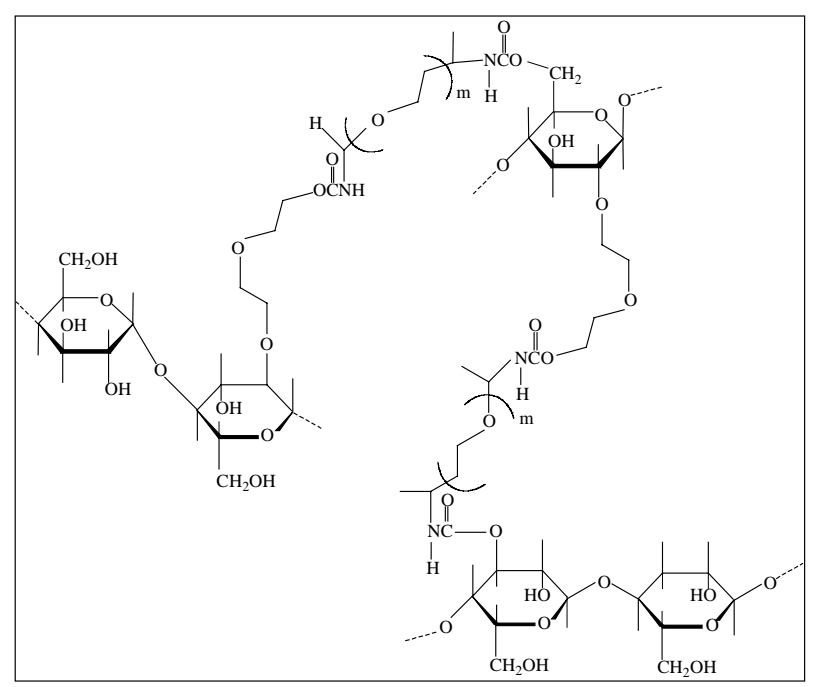

Figura 4. Esquema simplificado da estrutura de rede de hidroxietil celulose enxertada com diisocianato de poli(óxido de etileno). 


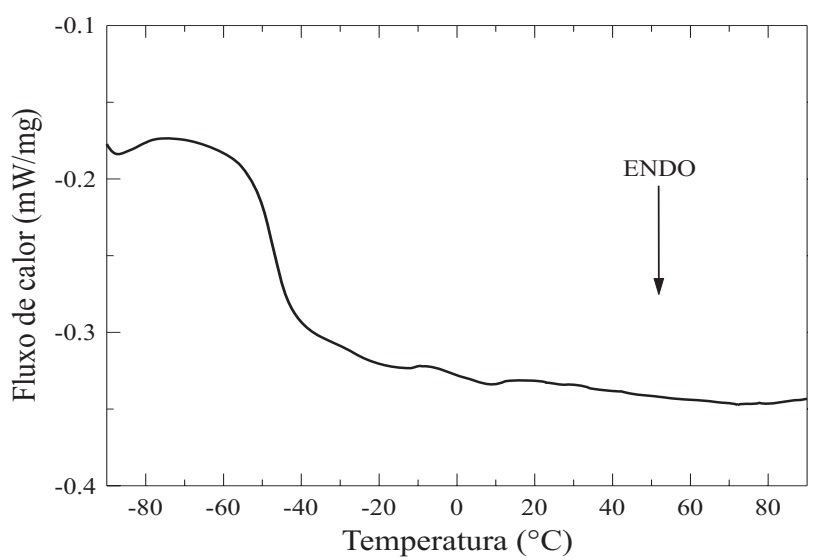

Figura 5. Análises de calorimetria diferencial exploratória de rede de hidroxietil celulose (QP4400) enxertada com diisocianato de poli(óxido de etileno) (DPOE).

Tabela 3. Valores da Tg para os filmes obtidos de HEC enxertada com diisocianatos de poli(óxido de etileno) (DPOE) e poli(óxido de propileno) (DPOP)

\begin{tabular}{lcc}
\hline \multicolumn{1}{c}{ Amostra } & \multicolumn{2}{c}{$\operatorname{Tg}\left({ }^{\circ} \mathrm{C}\right)$} \\
\cline { 2 - 3 } & DPOE & DPOP \\
\hline QP09H & -54 & -36 \\
QP300H & -47 & -35 \\
QP4400 & -48 & -35 \\
QP15MH & -52 & -33 \\
QP30MH & -52 & -34 \\
\hline
\end{tabular}

As curvas de DSC para todas as redes preparadas apresentam comportamento similar, sendo bem representada pela curva da amostra QP4400 enxertada com DPOE (Figura 5), onde pode-se observar a mudança de linha de base em torno de $-50^{\circ} \mathrm{C}$, ocorrência típica do fenômeno de transição vítrea. Nestas curvas não foram detectadas transições devidas à fusão, confirmando o caráter amorfo destas redes.

A comparação da temperatura de transição vítrea de uma série de redes de diferentes HEC, entrecruzadas com o mesmo tipo de diisocianato (Tabela 3), indicam que a $\mathrm{T}_{\mathrm{g}}$ é maior para os filmes obtidos pela grafitização de HEC com diisocianato de poli(óxido de propileno), em torno de $-35^{\circ} \mathrm{C}$, enquanto para as HEC enxertadas com poli(óxido de etileno), a transição ocorre em torno de $-50^{\circ} \mathrm{C}$.

Os valores obtidos para as temperaturas de transição vítrea são conseqüência da introdução dos isocianatos oligoméricos e da correspondente reticulação, uma vez que as amostras de HEC não apresentam esta característica. A temperatura de transição vítrea do material é afetada também pelo

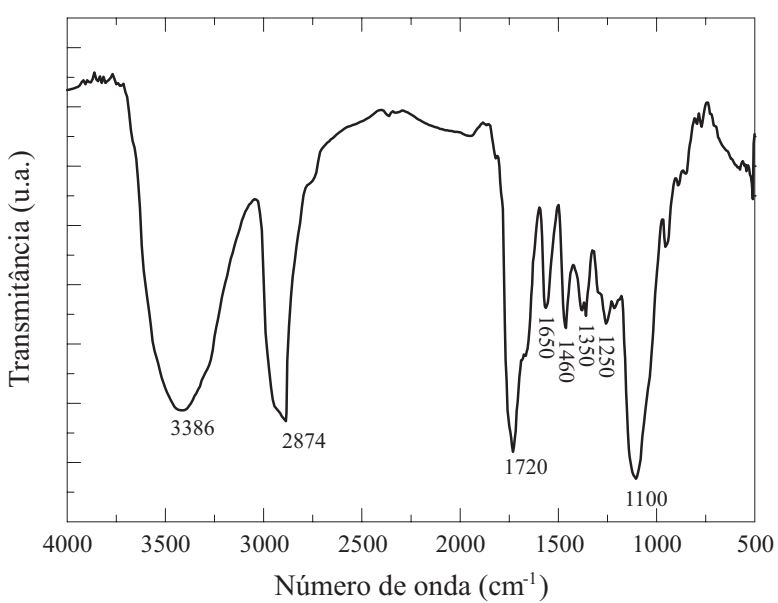

Figura 6. Espectro no infravermelho de hidroxietil celulose QP4400 enxertada com diisocianato de poli(óxido de etileno).

Tabela 4. Número de onda relativos às bandas características das vibrações das ligações presentes nas redes de hidroxietil celulose com DPOE.

\begin{tabular}{lc}
\hline \multicolumn{1}{c}{ Atribuição } & $\begin{array}{c}\text { Número de Onda } \\
\left(\mathbf{c m}^{-1}\right)\end{array}$ \\
\hline Deformação axial $\mathrm{NH}+\mathrm{OH}$ & 3350 (banda larga) \\
Deformação axial $\mathrm{CH}_{2}$ & 2860,2920 \\
Deformação axial C=O uretana & 1720 \\
Deformação axial C=O uréia & 1650 \\
Deformação angular de $\mathrm{CH}_{2}$ & 1460 \\
Deformação axial de CN & 1250 \\
Deformação axial C-O-C & 1100 \\
\hline
\end{tabular}

tipo de isocianato agregado à cadeia do polissacarídeo. A estrutura mais ramificada, contendo o grupo lateral metila, do poli(óxido de propileno) provoca um aumento de $15^{\circ} \mathrm{C}$ na temperatura de transição vítrea dos derivados, em relação as amostras de HEC enxertadas com poli(óxido de etileno). Esta tendência já foi observada em redes preparadas a partir de oligoéteres e poliisocianatos simples ${ }^{[2]}$.

A análise dos espectros na região do infravermelho de HEC entrecruzada com diisocianatos de poli(óxido de etileno) (Figura 6) permite classificar as bandas observadas (Tabela 4). A banda relativa à carbonila das funções uretana pode ser observada em $1720 \mathrm{~cm}^{-1}$; o pequeno ombro presente em $1650 \mathrm{~cm}^{-1}$ é devido à carbonila de uréia, presente nos espectros é proveniente do isocianato utilizado, já que este não foi submetido a nenhum procedimento adicional de purificação, a qual deve possuir algumas estruturas 
diméricas como conseqüência de condensação entre $\mathrm{NCO}$ terminais e grupos $\mathrm{NH}_{2}$.

A presença de banda de absorção em torno de $300 \mathrm{~nm}$, nos espectros registrados na região do ultravioleta para os filmes obtidos a partir das redes é relativa à absorção do grupo cromóforo RCONHR, confirmando a obtenção das funções uretana. Na região visível os filmes são transparentes, o que é desejável do ponto de vista de sua possível aplicação em dispositivos eletrocrômicos.

$\mathrm{O}$ experimento de difração de raios-X apontou uma banda de absorção centrada em ângulo de Bragg próximo a $2 \theta=20^{\circ}$ (Figura 3 ) que é característica da HEC e derivados da celulose, confirmando também a predominância do material amorfo.

\section{Conclusões}

A análise dos espectros de IR e UV confirmou a formação de redes de hidroxietil celulose com diisocianatos de poli(óxido de etileno) e poli(óxido de propileno). As amostras na forma de filmes apresentaram a temperatura de transição vítrea muito abaixo da temperatura ambiente. Este é um fator importante do ponto de vista de transporte iônico (e portanto da condutividade) nestes materiais, que depende da mobilidade dos segmentos de cadeias de poliéteres. $\mathrm{O}$ valor desta temperatura de transição vítrea é também função do tipo de isocianato utilizado na enxertia, sendo que para as redes formadas com HEC e os diisocianatos de poli(óxido de etileno) este valor fica em torno de $-50^{\circ} \mathrm{C}$, enquanto para as redes formadas com HEC e diisocianatos de poli(óxido de propileno) fica em torno de $-35^{\circ} \mathrm{C}$.

\section{Agradecimentos}

Os autores agradecem a FAPESP pela bolsa concedida para Anelise M. Regiani, a UNION CARBIDE do Brasil pelas amostras de HEC e ao Programa USP/COFECUB, pelo suporte financeiro desta colaboração

\section{Referências Bibliográficas}

1. Zoppi, R. A.; Nunes, S. P. - Polímeros: Ciência e Tecnologia, № 4, p. 27-36 (1997).
2. Le Nest, J. F.; Gandini, A.; Cheradame, H. - British Polymer Journal, 20, p. 253-268 (1988).

3. Sircar, A. K.; Weissman, P. T.; Kumar B. Thermochimica Acta, 26, p. 281-299 (1993).

4. Le Nest, J. F.; Gandini, A.; Schoenenberger C. Trends in Polym. Sci., 2, p. $432-437$ (1994).

5. Fengel, D. \& Wegener, G. - "Wood - Chemistry, Ultrastructure, Reactions", Walter de Gruyter, Berlin (1984).

6. Schoenenberger, C.; Le Nest, J. F.; Gandini A. - Electrochimica Acta, 40, n. 13-14, p. 22812284 (1995).

7. Moralez, P. V.; LeNest, J. F.; Gandini A. Electrochimica Acta, 43, n. 10-11, p. 12751279 (1998).

8. Clemett, C. J. - Analytical Chemistry, 45, 1, p. 186-188 (1973).

9. DeMember, J. R.; Taylor, L. D.; Trummer, S.; Rubin, L. E.; Chiklis C. K. - J. Appl. Polym. Sci., 21, p. 621-627 (1977).

10. Brown, W.; Henley, O.; Öhman. J. - Makromol. Chimie, 64, p. 49-67 (1963).

11. Kaloustian, J.; Pauli, A. M.; Pastor J. - J. Thermal Appl. Analysis, 48, 791-804 (1997).

12. Standard Test Method for Isocyanate Groups in Urethane Materials Prepolymers. /ASTM D $2572-87 /$.

13. Schoeneberger C. - Tese de Doutorado, Institut National Polytechnique de Grenoble, (1994).

14. Le Nest, J. F.; Callens, S.; Gandini, A.; Armand M. - Electrochim. Acta, 37, 9, p. 1585-1588 (1992).

15. Le Nest, J. F.; Gandini, A.; Xu, L.; Schoeneberger C. - Polym. Adv. Technol., 4, p. 92- 98 (1993).

16. UNION CARBIDE PRODUTOS QUÍMICOS LTDA. "Cellozise, hidroxietil celulose HEC", Union Carbide Corporation, U.S.A., (1981) (catálogo).

Recebido: 02/02/99 Aprovado: 03/09/99 